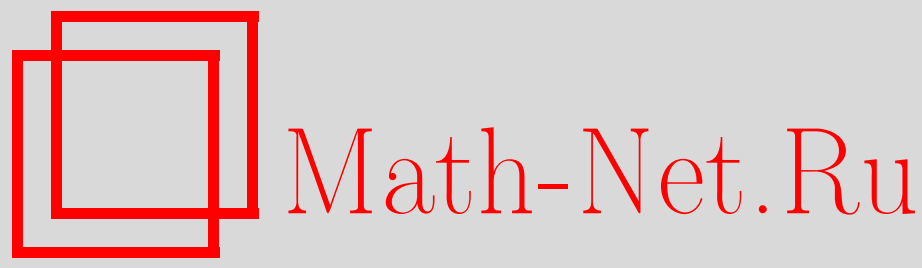

М. Э. Муминов, О положительности двухчастичного гамильтониана на решетке, ТМФ, 2007, том 153, номер 3, 381-387

DOI: https://doi.org/10.4213/tmf6143

Использование Общероссийского математического портала Math-Net.Ru подразумевает, что вы прочитали и согласны с пользовательским соглашением http://www.mathnet.ru/rus/agreement

Параметры загрузки:

IP: 54.209 .52 .79

26 апреля 2023 г., 18:07:58

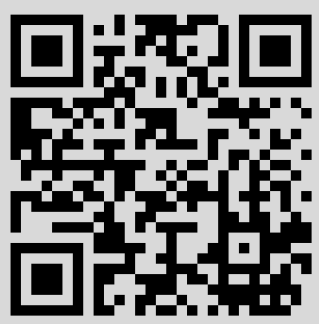




\title{
О ПОЛОЖИТЕЛЬНОСТИ ДВУХЧАСТИЧНОГО ГАМИЛЬТОНИАНА НА РЕШЕТКЕ
}

\begin{abstract}
Рассмотрен двухчастичный гамильтониан на $d$-мерной решетке $\mathbb{Z}^{d}$. Найдено достаточное условие положительности семейства операторов $h(k)$, появляющихся после "выделения центра масс" системы двух частиц, зависящих от значений полного квазиимпульса $k \in T^{d}$ ( $T^{d}-d$-мерный тор). На основании полученного результата показано существование положительных собственных значений оператора $h(k)$ при ненулевых $k \in T^{d}$.
\end{abstract}

Ключевые слова: двухчастичный гамильтониан на решетке, виртуальный уровень, регулярная точка, положительный оператор, дискретный спектр.

\section{1. ВВЕДЕНИЕ И ФОРМУЛИРОВКА ОСНОВНЫХ РЕЗУЛЬТАТОВ}

При получении двухчастичного оператора Шредингера (в непрерывном случае) из полного гамильтониана можно выделить энергию движения центра масс так, что одночастичные "связанные состояния" оказываются собственными векторами оператора энергии с отделенным полным импульсом (при этом такой оператор фактически не зависит от значений полного импульса) [1]. На решетке "выделению центра масс" системы отвечает реализация гамильтониана как "расслоенного оператора", т.е. прямого интеграла семейства $h(k), k \in T^{d}$ ( $T^{d}-d$-мерный тор), операторов энергии двух частиц, где $k$-значение полного квазиимпульса [2], [3].

Решетчатые двухчастичные гамильтонианы иследованы в работах [4]-[6]. В работе [4] подробно рассмотрена задача о спектре двухчастичных связанных состояний трансфер-матриц широкого класса гиббсовских полей на решетке в высокотемпературной области $(t \gg 1)$, в работе [5] показано, что при некоторых значениях полного квазиимпульса системы появляются уровни связанных состояний, отстоящие от непрерывного спектра на определенное расстояние. Спектральные свойства двухчастичного оператора, зависящие от полного квазиимпульса, изучены в работе [6].

В недавней работе [3] получен замечательный результат, который существенно отличается от непрерывного случая: если оператор $h(0)$ имеет виртуальный уровень

${ }^{*}$ Самаркандский государственный университет, Самарканд, Узбекистан. E-mail: mmuminov@mail.ru 
на левом крае существенного спектра (см. далее определение 2), то дискретный спектр оператора $h(k)$, лежащий левее существенного спектра, является непустым при всех $k \in T^{d} \backslash\{0\}$.

Заметим, что существенный спектр многочастичных операторов Шредингера тесно связан с множеством $\bigcup_{k \in T^{d}} \sigma(h(k))$, где $\sigma(h(k))$ - спектр двухчастичного оператора Шредингера $h(k)$.

В настоящей работе найдено такое условие для параметра, задающего оператор $h(k)$, что из неотрицательности $h(0)$ следует неотрицательность $h(k)$ при всех $k \in T^{d} \backslash\{0\}$. При этом если слева от нуля отсутствуют связанные состояния оператора $h(0)$, то для всех полных квазиимпульсов $k$ оператора $h(k)$ также отсутствуют связанные состояния слева от нуля. На примерах показано, что найденное условие является существенным для неотрицательности $h(k)$. Как следствие этого результата, наложив дополнительные требования в теореме 2 работы [3], мы доказали, что дискретный спектр $h(k)$, лежащий левее существенного спектра $\sigma_{\mathrm{ess}}(h(k))$, не пуст и содержится в полуинтервале $\left[0, \varepsilon_{\min }(k)\right), \varepsilon_{\min }(k)=\inf \sigma_{\operatorname{ess}}(h(k))$.

Пусть $\mathbb{Z}^{d}-d$-мерная целочисленная решетка, $\left(\mathbb{Z}^{d}\right)^{2}=\mathbb{Z}^{d} \times \mathbb{Z}^{d}$ - декартова степень $\mathbb{Z}^{d}$ и $l_{2}\left(\left(\mathbb{Z}^{d}\right)^{2}\right)$ - гильбертово пространство квадратично-суммируемых функций, определенных на $\left(\mathbb{Z}^{d}\right)^{2}$.

В координатном представлении гамильтониан системы двух произвольных частиц с парным короткодействующим потенциалом $\hat{v}(\cdot)$ взаимодействия на $d$-мерной решетке, действующий в пространстве $l_{2}\left(\left(\mathbb{Z}^{d}\right)^{2}\right)$, имеет вид

$$
\hat{h}=\hat{h}_{0}-\hat{v}
$$

где $\hat{h}_{0}$ и $\hat{v}$ задаются формулами

$$
\begin{aligned}
\left(\hat{h}_{0} \hat{\psi}\right)\left(n_{1}, n_{2}\right) & =\sum_{s \in \mathbb{Z}^{d}}\left[\hat{\varepsilon}_{1}(s) \hat{\psi}\left(n_{1}+s, n_{2}\right)+\hat{\varepsilon}_{2}(s) \hat{\psi}\left(n_{1}, n_{2}+s\right)\right], \\
(\hat{v} \hat{\psi})\left(n_{1}, n_{2}\right) & =\hat{v}\left(n_{1}-n_{2}\right)(\hat{\psi})\left(n_{1}, n_{2}\right) .
\end{aligned}
$$

Здесь $\hat{\varepsilon}_{1}(s)$ и $\hat{\varepsilon}_{2}(s)$ - вещественнозначные четные функции, описывающие перенос частицы с узла на соседний узел, функция $\hat{v}(\cdot) \in l_{2}\left(\mathbb{Z}^{d}\right)$.

Мы предполагаем, что функции $\hat{\varepsilon}_{1}(\cdot)$ и $\hat{\varepsilon}_{2}(\cdot)$ неотрицательные и выполнено условие

$$
\sum_{s \in \mathbb{Z}^{d}}\left|\hat{\varepsilon}_{j}(s)\right|<\infty, \quad j=1,2 .
$$

Пусть $T=(-\pi, \pi], L_{2}\left(T^{d}\right)$ - гильбертово пространство всех квадратично-интегрируемых функций, определенных на $T^{d}$. Используя стандартное преобразование Фурье [3], [7]

$$
\Im: L_{2}\left(\left(T^{d}\right)^{2}\right) \rightarrow l_{2}\left(\left(\mathbb{Z}^{d}\right)^{2}\right)
$$

и разложение в прямой интеграл, мы сводим изучение спектральных свойств оператора $\hat{h}$ к исследованию спектральных свойств семейства самосопряженных операторов $h(k), k \in T^{d}$, действующих в гильбертовом пространстве $L_{2}\left(T^{d}\right)$ по формуле

$$
h(k)=h_{0}(k)-\mathbf{v} .
$$


Здесь $h_{0}(k)$ - оператор умножения на функцию

$$
\mathcal{E}_{k}(p)=\varepsilon_{1}(p)+\varepsilon_{2}(p+k)
$$

$\mathbf{v}$ - интегральный оператор с ядром $v(p-s)$, где $v(\cdot)$ и $\varepsilon_{j}(\cdot)$ - преобразования Фурье функций $\hat{v}(\cdot)$ и $\hat{\varepsilon}_{j}(\cdot)$, соответственно:

$$
v(p)=\frac{1}{(2 \pi)^{d / 2}} \sum_{s \in \mathbb{Z}^{d}} \hat{v}(s) e^{i(p, s)}, \quad \varepsilon_{j}(p)=\frac{1}{(2 \pi)^{d / 2}} \sum_{s \in \mathbb{Z}^{d}} \hat{\varepsilon}_{j}(s) e^{i(p, s)}, \quad j=1,2 .
$$

ПрЕДПОЛОжЕНИЕ. Предположим, что $\varepsilon_{j}(p), j=1,2$, - непрерывные (периодические) вещественнозначные функции на $T^{d}, d \geqslant 3$, имеющие единственную невырожденную точку минимума в центре координат, причем

$$
\liminf _{|p| \rightarrow 0} \frac{\varepsilon_{j}(p)-\varepsilon_{j}(0)}{|p|^{2}}>0
$$

Кроме того, пусть $v(\cdot)$ - непрерывная функция на $T^{d}$ такая, что

$$
v(p)=\overline{v(-p)}, \quad p \in T^{d}, \quad d \geqslant 3 .
$$

Отметим, что из теоремы Вейля о существенном спектре [8] следует, что существенный спектр $\sigma_{\text {ess }}(h(k))$ оператора $h(k)$ не меняется при компактом возмущении оператора $\mathbf{v}$ и совпадает со спектром невозмущенного оператора $h_{0}(k)$. При этом $\sigma_{\text {ess }}(h(k))$ совпадает с областью значений функции $\mathcal{E}_{k}(\cdot)$, т.е.

$$
\sigma_{\mathrm{ess}}(h(k))=\sigma\left(h_{0}(k)\right)=\left[\varepsilon_{\min }(k), \varepsilon_{\max }(k)\right],
$$

где $\varepsilon_{\min }(k)=\min _{p} \mathcal{E}_{k}(p), \varepsilon_{\max }(k)=\max _{p} \mathcal{E}_{k}(p)$. Из сделанного выше предположения следует, что $\varepsilon_{\min }(k)>\varepsilon_{\min }(0)=0$ при $k \in T^{d}$.

Пусть $C\left(T^{d}\right)$ - банахово пространство непрерывных (периодических) функций на $T^{d}, G(\lambda), \lambda \leqslant \mathcal{E}_{0}(0),-$ интегральный оператор с ядром (Бирмана-Швингера)

$$
G(p, q ; \lambda)=(2 \pi)^{-d / 2} v(p-q)\left(\mathcal{E}_{0}(p)-\lambda\right)^{-1}, \quad p, q \in T^{d} .
$$

Следующие возможные случаи для собственного значения оператора $G(\lambda)$ при $\lambda=\mathcal{E}_{0}(0)$ детально обсуждены в работе [3].

СлУчАЙ 1. Число -1 не является собственным значением оператора $G\left(\mathcal{E}_{0}(0)\right)$, т.e.

$$
\operatorname{dim} \operatorname{Ker}\left(h(0)-\mathcal{E}_{0}(0) I\right)=\operatorname{dim} \operatorname{Ker}\left(G\left(\mathcal{E}_{0}(0)\right)+I\right)=0 .
$$

СлУчАЙ 2. Число -1 является простым собственным значением оператора $G\left(\mathcal{E}_{0}(0)\right)$, и соответствующая собственная функция $\psi$ удовлетворяет условию

$$
\frac{\psi(0)}{\mathcal{E}_{0}(p)-\mathcal{E}_{0}(0)} \notin L_{2}\left(T^{d}\right)
$$

T.e.

$$
\operatorname{dim} \operatorname{Ker}\left(h(0)-\mathcal{E}_{0}(0) I\right)=0, \quad \operatorname{dim} \operatorname{Ker}\left(G\left(\mathcal{E}_{0}(0)\right)+I\right)=1 .
$$


СлУчАй 3. Число -1 является кратным собственным значением оператора $G\left(\mathcal{E}_{0}(0)\right)$, и одна из соответствующих собственных функций $\psi$ удовлетворяет условию

$$
\frac{\psi(0)}{\mathcal{E}_{0}(p)-\mathcal{E}_{0}(0)} \notin L_{2}\left(T^{d}\right),
$$

т.е.

$\operatorname{dim} \operatorname{Ker}\left(h(0)-\mathcal{E}_{0}(0) I\right) \geqslant 2, \quad \operatorname{dim} \operatorname{Ker}\left(h(0)-\mathcal{E}_{0}(0) I\right) \geqslant \operatorname{dim} \operatorname{Ker}\left(G\left(\mathcal{E}_{0}(0)\right)+I\right)+1$.

СлУчАй 4. Число -1 является кратным собственным значением оператора $G\left(\mathcal{E}_{0}(0)\right)$ и

$$
\operatorname{dim} \operatorname{Ker}\left(h(0)-\mathcal{E}_{0}(0) I\right)+2 \leqslant \operatorname{dim} \operatorname{Ker}\left(G\left(\mathcal{E}_{0}(0)\right)+I\right) .
$$

ОПРЕДЕЛЕНИЕ 1 [9]. Если имеет место случай 1, нуль называется регулярной точкой оператора $h(0)$.

ТЕОРема 1. Пусть функиии $\varepsilon_{1}(\cdot)$ и $\varepsilon_{2}(\cdot)$ являются линейно зависимыми. Если $h(0) \geqslant 0$, mо $h(k) \geqslant 0$ для всех $k \in T^{d}$. Дополнительно, если нуль - регулярная mочка оператора $h(0)$, то $(h(k) f, f)>0$ для всех $f \neq 0 u k \in T^{d}$.

ЗАмЕЧАниЕ. Условия теоремы 1 являются существенными для положительности $h(k)$ при $k \in T^{3}$ (см. пример 1). При выполнении условий теоремы 1 из того, что $h(k) \geqslant 0$ для некоторого $k \in T^{3}$, не всегда вытекает, что $h(0) \geqslant 0$ (см. пример 2).

ОПРЕДЕЛЕНИЕ 2. Пусть $d=3,4$. Если имеет место один из случаев 2-4, то говорят, что оператор $h(0)$ имеет виртуальный уровень в нуле (на левом крае существенного спектра).

Нам понадобится следующая теорема, доказанная в работе [3].

Теорема 2. Пусть $d \geqslant 3$, выполнено указанное выше предположение $u \varepsilon_{1}(\cdot)$, $\varepsilon_{2}(\cdot)$ - условно отрицательно определенные функиии ${ }^{1)}$, дифберениируемые до второго порядка. Предположим, что $h(0)$ имеет виртуальный уровень (на левом крае существенного спектра). Тогда для каждого $k \in T^{d} \backslash\{0\}$ дискретный спектр оператора $h(k)$, лежсащий левее $\varepsilon_{\min }(k)$, представляет собой непустое множество.

Пользуясь теоремой 1 , из теоремы 2 получим

СлеДСТвИЕ. Пусть выполнены условия теоремы 2. Пусть, кроме того, функиии $\varepsilon_{1}(\cdot), \varepsilon_{2}(\cdot)$ являются линейно зависимыми и $h(0) \geqslant 0$. Тогда для каждого $k \in T^{d} \backslash\{0\}$ дискретный спектр оператора $h(k)$, лежащий левее существенного спектра, есть непустое множество, содержащееся в полуинтервале $\left[0, \varepsilon_{\min }(k)\right)$.

\footnotetext{
1) Функция $\varepsilon(\cdot)$ называется условно отрицательно определенной (см. [8]), если $\varepsilon(p)=\overline{\varepsilon(p)}$ и для каждого натурального числа $n$ и для всех $p_{1}, \ldots, p_{n} \in T^{d}, z=\left(z_{1}, \ldots, z_{n}\right) \in \mathbb{C}^{n}($ где $\mathbb{C}-$ комплексная плоскость) выполнено неравенство
}

$$
\sum_{i, j=1}^{n} \varepsilon\left(p_{i}-p_{j}\right) z_{i} \bar{z}_{j} \leqslant 0 .
$$


В качестве примера функций $\varepsilon_{1}(\cdot)$ и $\varepsilon_{2}(\cdot)$, удовлетворяющих условиям следствия, можно рассмотреть $\varepsilon_{j}(\cdot)=\varepsilon(\cdot) / 2 m_{j}$, где $m_{j}$ - масса частицы, $j=1,2$, и $\varepsilon(p)=$ $2 \sum_{k=1}^{d}\left(1-\cos p_{k}\right)$. В этом случае преобразование Фурье $\hat{\varepsilon}(\cdot)$ функции $\varepsilon(\cdot)$ имеет вид

$$
\hat{\varepsilon}(s)=\left\{\begin{array}{rll}
2 d & \text { при } \quad s=0, \\
-1 & \text { при } \quad|s|=1 \\
0 & \text { в остальных случаях. }
\end{array}\right.
$$

\section{2. ДОКАЗАТЕЛЬСТВО ОСНОВНЫХ РЕЗУЛЬТАТОВ}

Доказательство теоремы 1. Предположим, что $h(0)$ является неотрицательным оператором, т.е. $h(0) \geqslant 0$. Оператор $h(k)$ запишем в виде

$$
h(k)=\frac{m_{2}}{m_{1}+m_{2}} h(0)+\frac{m_{1}}{m_{1}+m_{2}} U_{k} h(0) U_{k}^{-1}, \quad k \in T^{d} \backslash\{0\},
$$

где $U_{k}: L_{2}\left(T^{d}\right) \rightarrow L_{2}\left(T^{d}\right)$ - унитарный оператор, определенный формулой $\left(U_{k} f\right)(q)=$ $f(k+q), f \in L_{2}\left(T^{d}\right)$. Отсюда и из неотрицательности $h(0)$ имеем

$$
(h(k) f, f)=\frac{m_{2}}{m_{1}+m_{2}}(h(0) f, f)+\frac{m_{1}}{m_{1}+m_{2}}\left(U_{k} h(0) U_{k}^{-1} f, f\right) \geqslant 0
$$

для каждого $f \in L_{2}\left(T^{d}\right)$.

Пусть нуль является регулярной точкой оператора $h(0)$. Тогда из $h(0) f \neq 0$ при $f \neq 0$ имеем $\left.(h(0) f, f)=\left([h(0)]^{1 / 2} f, h(0)\right]^{1 / 2} f\right)>0$. Поэтому из (2.1) вытекает, что $(h(k) f, f)>0$ для всех $k \neq 0$ и $f \neq 0$. Теорема доказана.

Пример 1. Рассмотрим случай, когда потенциал $\hat{v}$ рассматриваемой системы определен как парный контактный потенциал $\hat{v}(\cdot)=\mu \delta_{n_{1} n_{2}}$, где $\mu>0$ - энергия взаимодействия двух частиц, $\delta_{n_{1} n_{2}}-$ символ Кронекера. В этом случае $h_{\mu}(k)=h(k)$ действует по формуле

$$
\left(h_{\mu}(k) f\right)(p)=\left[\varepsilon_{1}(p)+\varepsilon_{2}(p-k)\right] f(p)-\frac{\mu}{(2 \pi)^{d / 2}} \int f(s) d s .
$$

Пусть $d=3$ и

$$
\varepsilon_{1}(p)=\sum_{i=1}^{3}\left(1-\cos p_{i}\right) \cos ^{4} p_{i}, \quad \varepsilon_{2}(p)=\sum_{i=1}^{3} \sin ^{4} p_{i} .
$$

Функции $\varepsilon_{1}(\cdot), \varepsilon_{2}(\cdot)$ являются линейно независимыми.

Легко проверить (см. [7]), что число $z \in \mathbb{C} \backslash \sigma_{\text {cont }}\left(h_{\mu}(k)\right)$ является собственным значением оператора $h_{\mu}(k)$ тогда и только тогда, когда

$$
\Delta_{\mu}(k ; z)=1-\frac{\mu}{(2 \pi)^{3 / 2}} \int_{T^{3}} \frac{d s}{\varepsilon_{1}(s)+\varepsilon_{2}(s+k)-z}=0 .
$$

Отметим, что интеграл

$$
\int_{T^{3}} \frac{d s}{\varepsilon_{1}(s)+\varepsilon_{2}(s+k)-z}
$$

4 Теоретическая и математическая физика, т. 153, № 3, 2007 г. 
существует при $z=0, k \in T^{3}$. Пусть

$$
\mu_{0}=(2 \pi)^{3 / 2}\left(\int_{T^{3}} \frac{d s}{\varepsilon_{1}(s)+\varepsilon_{2}(s)}\right)^{-1} .
$$

Тогда $\Delta_{\mu}(0 ; z) \geqslant 0$ при $\mu \in\left(0, \mu_{0}\right]$ и $z \leqslant 0$. Следовательно, оператор $h_{\mu}(0)$ не имеет отрицательных собственных значений при $\mu \in\left(0, \mu_{0}\right]$. Отсюда и из неравенства $\varepsilon_{\min }(k) \geqslant 0$ получим $h(0) \geqslant 0$.

Покажем, что $h_{\mu}(k), \mu \in\left(0, \mu_{0}\right]$, не является положительным оператором при $k^{0}=(\pi / 2, \pi / 2, \pi / 2)$.

Используя равенство

$$
\varepsilon_{1}(p)+\varepsilon_{2}\left(p-k^{0}\right)=\sum_{i=1}^{3}\left(1-\cos p_{i}\right) \cos ^{4} p_{i}+\sum_{i=1}^{3} \sin ^{4}\left(\frac{\pi}{2}-p_{i}\right)=\sum_{i=1}^{3}\left(2-\cos p_{i}\right) \cos ^{4} p_{i}
$$

и учитывая периодичность функции $\varepsilon_{1}(p)+\varepsilon_{2}\left(p-k^{0}\right)$, получим равенство

$$
\int_{T^{3}} \frac{d s}{\varepsilon_{1}(s)+\varepsilon_{2}\left(s+k^{0}\right)-z}=\int_{T^{3}} \frac{d p_{1} d p_{2} d p_{3}}{\sum_{i=1}^{3}\left[2-\cos \left(\pi / 2+p_{i}\right)\right] \sin ^{4} p_{i}-z}, \quad z<0 .
$$

Согласно этому равенству для каждого фиксированного $\mu>0$

$$
\lim _{z \rightarrow-0} \Delta_{\mu}\left(k^{0} ; z\right)=-\infty
$$

Функция $\Delta_{\mu}^{0}\left(k^{0} ; \cdot\right)$ монотонна и непрерывна на луче $(-\infty, 0), \lim _{z \rightarrow-\infty} \Delta_{\mu}\left(k^{0} ; z\right)=1$, поэтому $\Delta_{\mu}\left(k^{0} ; z_{0}\right)=0$ в некоторой точке $z_{0} \in(-\infty, 0)$, т.е. оператор $h\left(k^{0}\right)$ имеет отрицательное собственное значение $z_{0}$.

ПримеР 2. Рассмотрим случай, когда в (2.2)

$$
\varepsilon_{1}(p)=\varepsilon_{2}(p)=\sum_{i=1}^{3}\left(1-\cos p_{i}\right)
$$

Пусть $\bar{k}=\left(\bar{k}_{1}, \bar{k}_{2}, \bar{k}_{3}\right) \in T^{3} \backslash\{0\}$ и $\bar{\mu}>0$ таково, что

$$
\bar{\mu} \int_{T^{3}} \frac{d s}{\mathcal{E}_{\bar{k}}(s)}=(2 \pi)^{3 / 2} .
$$

Тогда $h_{\bar{\mu}}\left(k^{0}\right) \geqslant 0$. Покажем, что $h_{\bar{\mu}}(0)$ не является положительным оператором. Для этого достаточно показать существование отрицательного собственного значения оператора $h_{\bar{\mu}}(0)$.

Учитывая элементарное равенство

$$
\varepsilon_{1}(p)+\varepsilon_{2}(p-k)=\sum_{i=1}^{3}\left[2-\sqrt{2+2 \cos k_{i}} \cos \left(p_{i}-\alpha\left(k_{i}\right)\right)\right], \quad k=\left(k_{1}, k_{2}, k_{3}\right),
$$


где $\alpha\left(k_{i}\right)=\arcsin \frac{\sin k_{i}}{\sqrt{2+2 \cos k_{i}}}$, из периодичности функции $\varepsilon_{k}(\cdot)$ получаем, что

$$
\begin{aligned}
\Delta_{\bar{\mu}}(\bar{k} ; 0) & =1-\frac{\bar{\mu}}{(2 \pi)^{3 / 2}} \int_{T^{3}} \frac{d p_{1} d p_{2} d p_{3}}{\sum_{i=1}^{3}\left[2-\sqrt{2+2 \cos \bar{k}_{i}} \cos \left(p_{i}-\alpha\left(\bar{k}_{i}\right)\right)\right]}= \\
& =1-\frac{\bar{\mu}}{(2 \pi)^{3 / 2}} \int_{T^{3}} \frac{d s_{1} d s_{2} d s_{3}}{\sum_{i=1}^{3}\left[2-\sqrt{2+2 \cos \bar{k}_{i}} \cos s_{i}\right]} .
\end{aligned}
$$

Отсюда и из неравенства

$$
\int_{-\pi}^{\pi} \frac{d s}{a^{2}+\sqrt{2+2 \cos \bar{k}_{i}} \cos s} \leqslant \int_{-\pi}^{\pi} \frac{d s}{a^{2}+2 \cos s},
$$

верного при $a \neq 0$, имеем

$$
0=\Delta_{\bar{\mu}}(\bar{k} ; 0)>1-\frac{\bar{\mu}}{(2 \pi)^{3 / 2}} \int_{T^{3}} \frac{d s}{\varepsilon_{1}(s)+\varepsilon_{2}(s)}=\Delta_{\bar{\mu}}(0 ; 0) .
$$

Из условия $\lim _{z \rightarrow-\infty} \Delta_{\bar{\mu}}(0 ; z)=1$ и непрерывности функции $\Delta_{\bar{\mu}}(0 ; \cdot)$ на $(-\infty, 0]$ получаем, что $\Delta_{\bar{\mu}}\left(0 ; z_{0}\right)=0$ для некоторого $z_{0} \in(-\infty, 0)$, т.е. оператор $h(0)$ имеет отрицательное собственное значение $z_{0}$.

ДокАЗАТЕЛЬСтво СЛЕДствия. Из теоремы 2 следует, что дискретный спектр оператора $h(k)$, лежащий левее $\varepsilon_{\min }(k)$, является непустым, т.е.

$$
\sigma_{\text {disc }}(h(k)) \cap\left(-\infty, \varepsilon_{\min }(k)\right) \neq \varnothing .
$$

По теореме $1 h(k) \geqslant 0$ и поэтому $\sigma_{\text {disc }}(h(k)) \cap\left(-\infty, \varepsilon_{\min }(k)\right) \subset\left[0, \varepsilon_{\min }(k)\right)$.

Благодарности. Работа выполнена при частичной поддержке ФПФИ АН РУз (грант № 77-06).

\section{Список литературы}

[1] Л. Д. Фаддеев, Тр. МИАН, 69 (1963), 1-122.

[2] D. C. Mattis, Rev. Mod. Phys., 58 (1986), 361-379.

[3] S. Albeverio, S. N. Lakaev, K.A. Makarov, Z.I. Muminov, Commun. Math. Phys., 262 (2006), 91-115.

[4] Е. Л. Лакштанов, Р. А. Минлос, Функи. анализ и его прилож., 38:3 (2004), 52-69.

[5] Е. Л. Лакштанов, Р. А. Минлос, Функи. анализ и его прилож., 39:1 (2005), 39-55.

[6] P. A. Faria da Veiga, L. Ioriatti, M. O'Carroll, Phys. Rev. E, 66 (2002), 016130.

[7] С. Н. Лакаев, М.Э. Муминов, ТМФ, 135:3 (2003), 478-503.

[8] М. Рид, Б. Саймон, Методы современной математической физики. Т. 4. Анализ операторов, Мир, М., 1982.

[9] A. V. Sobolev, Commun. Math. Phys., 156 (1993), 101-126.

Поступила в редакцию 15.01.2007, после доработки 2.05.2007 